\title{
Moyal and tomographic probability representations for f-oscillator quantum states
}

\author{
Vladimir I. Man'ko, ${ }_{1}^{1}$ Giuseppe Marmo, ${ }^{2}$ and Francesco Zaccaria ${ }^{2}$ \\ ${ }^{1}$ P.N. Lebedev Physical Institute, Leninskii Prospect, 53, Moscow 119991 \\ Russia \\ ${ }^{2}$ Dipartimento di Scienze Fisiche, Università "Federico II" di Napoli and \\ Istituto Nazionale di Fisica Nucleare, Sezione di Napoli, Complesso \\ Universitario di Monte Sant Angelo, Via Cintia, I-80126 Napoli, Italy \\ e-mail: manko@sci.lebedev.ru
}

\begin{abstract}
States of nonlinear quantum oscillators (f-oscillators) are considered in the Weyl-Wigner-Moyal representation and the tomographic probability representation, where the states are described by standard probability distributions instead of wave functions or density matrices. The evolving integrals of motion for classical and quantum f-oscillators are found and the solution for the Liouville equation associated with the probability distribution on the phase space for this oscillator is obtained along with the solution of Moyal equation for quantum f-oscillator, which provide the solutions for partial case of f-nonlinearity existing in Kerr media. Nonlinear coherent states and the thermodynamics of nonlinear oscillators are studied.
\end{abstract}

keywords: coherent states, Wigner function, tomographic probability, deformed oscillator.

\section{Introduction}

The small vibrations of classical and quantum systems are usually described by linear harmonic oscillator model. Recently $[1,2]$ the notion of f-oscillators and corresponding nonlinear coherent states for the oscillator quantum domain was introduced (see also [3]). The f-oscillators are nonlinear oscillators with a specific kind of nonlinearity for which the frequency depends on the oscillation energy [4]. The known q-oscillators [5,6] are very particular cases of the nonlinear vibrations with exponential dependence of the frequency on the energy of the vibrations. The properties of the f-oscillators and use of these oscillators for constructing models of different phenomena are intensively discussed in the literature, see, e.g., [7-18]. 
The nonlinearity of f-oscillators provides a deformation mechanism creating quantum group structures. For example, the f-deformed Weyl systems corresponding to the deformation of the Heisenberg-Weyl group were studied in [19] and q-nonlinearity corresponds to q-deformed Heisenberg-Weyl group. Recently in quantum [20] and classical mechanics [21,22] the tomographic probability representation of system states was suggested. In classical statistical mechanics, the tomographic probability distribution (tomogram or symplectic tomogram) is the Radon transform [23] of the probability distribution of the system on its phase space which is also the standard probability distribution. In quantum mechanics, the tomogram is the Radon transform of the Wigner function [24] of quantum states. In Moyal approach [25], the quantum states are described by the Wigner function and obey the evolution equation which is similar to the classical Liouville equation. The Wigner function, which is the Weyl symbol [26] of the density operator $\rho$, can take negative values and, in view of this, it is not a probability distribution. It is called quasidistribution and contains complete information on quantum states. The symplectic tomogram also contains complete information on quantum state of a system but the tomogram is the measurable nonnegative probability distribution. The approach to describe the quantum states by the synplectic tomograms is called the tomographic probability representation of quantum mechanics and its properties are studied in different aspects in [27-34].

The aim of this paper is to find integrals of motion and to consider the behaviour of f-oscillators, both classical and quantum ones, in the tomographic probability representation. We study also quantum f-oscillators in the Moyal representation. We study the thermodynamics of q-oscillator in the case of small nonlinearity. Both the Weyl-Wigner-Moyal representation and the tomographic probability representation are examples of realizing the generic star-product scheme (see, e.g., [27,35-39]). In this context, the goal of the paper is also to associate the behaviour of quantum nonlinear oscillator with the star-product quantization framework. We point out that the electromagnetic-field vibrations in Kerr media are a particular example of nonlinear f-oscillations with nonlinearity function proportional to the field amplitude.

The paper is organized as follows.

In Sect. 2, the properties of f-oscillators are reviewed both in the classical and quantum domains. In Sect. 3, the Moyal approach and construction of Weyl symbols, including a deformed Wigner function, are discussed. In 
Sect. 4, the tomography of classical and quantum states based on applying integral Radon transform is presented. In Sect. 5, nonlinear coherent states are studied. In Sect. 6, the partition function of nonlinear oscillator is considered. Finally the prospects and conclusions are done in Sect. 7.

\section{2 f-oscillators and kinetic equation}

\subsection{Classical nonlinear oscillator}

The coordinate $x(t)$ of linear harmonic classical oscillator satisfies the equation of motion

$$
\ddot{x}+x=0,
$$

where for simplicity the mass and frequency of the oscillator are set to be equal to unity, $m=\omega=1$.

For complex amplitude $\alpha(t)==(1 / \sqrt{2})(x+i \dot{x})$ the equation of motion reads

$$
\dot{\alpha}=-i \alpha
$$

and its solutions have the form

$$
\alpha(t)=\alpha_{0} e^{-i t}
$$

with $\alpha_{0}$ being any initial complex amplitude. The energy of vibrations is the integral of motion

$$
E=|\alpha(t)|^{2}=\left|\alpha_{0}\right|^{2}=\frac{\dot{x}^{2}}{2}+\frac{x^{2}}{2} .
$$

Thus the vibration of linear harmonic oscillator has the important property - the frequency of the vibration does not depend on the vibration energy. Another formulation of the same property implies that the phase of the vibrating linear oscillator is insensitive to the absolute value of the amplitude of the vibration. The simplest nonlinearity of vibration just violates this property. Let us consider the generalization of (3) taking the equation of motion of the form

$$
\dot{\alpha}=-i\left(\alpha \alpha^{*}\right) \alpha .
$$

One can see that $\frac{d}{d t}\left(\alpha \alpha^{*}\right)=0$, and the solutions (3) become

$$
\alpha(t)=\alpha_{0} e^{-i\left|\alpha_{0}\right|^{2} t}
$$


Thus for cubic nonlinear equation (5) the frequency of the vibration depends quadratically on the amplitude of the vibrations. The generic form of the equation of motion can be written as

$$
\dot{\alpha}=-i \omega\left(\alpha \alpha^{*}\right) \alpha,
$$

where the frequency function $\omega(E)$ depends on energy (44). The nonlinearity of vibrations is coded by the function $\omega(E)$. This means that the solution of Eq. (7)

$$
\alpha(t)=\alpha_{0} e^{-i \omega\left(\alpha_{0} \alpha_{0}^{*}\right) t}
$$

corresponds to the kind of the nonlinearity of the equation. If one describes the nonlinear-oscillator motion by the Hamiltonian

$$
H=\alpha_{f}^{*} \alpha_{f},
$$

in which the variable $\alpha_{f}$ reads as

$$
\alpha_{f}=\alpha f\left(\alpha^{*} \alpha\right)
$$

the dependence of the frequency on the energy is related to the dependence on the energy of the real function $f(E)$.

In fact, from (10) one has

$$
\omega(E)=f(E)+E f^{\prime}(E) .
$$

We call the f-oscillator the classical oscillator with the Hamiltonian (9), where $\alpha_{f}$ is given by (10), thus the oscillator evolves as

$$
\alpha(t)=\alpha_{0} \exp \left\{-i t\left[f\left(\alpha_{0} \alpha_{0}^{*}\right)+\left(\alpha_{0} \alpha_{0}^{*}\right) f^{\prime}\left(\alpha_{0} \alpha_{0}^{*}\right)\right]\right\} .
$$

The Liouville equation for the probability distribution $f(q, p, t)$ on phasespace reads

$$
\frac{\partial f(q, p, t)}{\partial t}+\frac{\partial f(q, p, t)}{\partial q} \dot{q}+\frac{\partial f(q, p, t)}{\partial p} \dot{p}=0
$$

Since for our f-oscillator

$$
\dot{q}=p\left[f(E)+E f^{\prime}(E)\right], \quad \dot{p}=-q\left[f(E)+E f^{\prime}(E)\right],
$$

one has the Liouville equation of the form

$$
\frac{\partial f(q, p, t)}{\partial t}+\frac{\partial f(q, p, t)}{\partial q} p\left[f(E)+E f^{\prime}(E)\right]-\frac{\partial f(q, p, t)}{\partial p} q\left[f(E)+E f^{\prime}(E)\right]=0,
$$


where $E=\left(q^{2}+p^{2}\right) / 2=\left(q_{0}^{2}+p_{0}^{2}\right) / 2$.

The system of equations (14) has two time-dependent integrals of motion, following the solution (12) of equation of motion (7)

$$
\begin{aligned}
& p_{0}=p \cos [\omega(E) t]+q \sin [\omega(E) t], \\
& q_{0}=-p \sin [\omega(E) t]+q \cos [\omega(E) t] .
\end{aligned}
$$

For a linear oscillator, $f=1$ and the integrals of motion have the form [40]

$$
p_{0}=p \cos t+q \sin t, \quad q_{0}=-p \sin t+q \cos t
$$

and their dependence on complex amplitude reads

$$
\alpha_{0}=\frac{q_{0}+i p_{0}}{\sqrt{2}}=\frac{q+i p}{\sqrt{2}} e^{i t} .
$$

For f-oscillator, one has the complex integral of motion generalizing the above invariant

$$
\alpha_{0}=\frac{q+i p}{\sqrt{2}} \exp \left[i \omega\left(\frac{q^{2}+p^{2}}{2}\right) t\right] .
$$

In terms of the integrals of motion, the solution of Liouville equation reads

$$
f(q, p, t)=f_{0}\left(q_{0}(t), p_{0}(t)\right)
$$

where $f_{0}(q, p)$ is the probability distribution given at time $t=0$. Thus, given the initial distribution $f_{0}(q, p)$ the form of the solution of the kinetic equation (15) is

$$
\begin{aligned}
f(q, p, t)= & f_{0}\left\{\left[-p \sin \omega\left(\frac{q^{2}+p^{2}}{2}\right) t+q \cos \omega\left(\frac{q^{2}+p^{2}}{2}\right) t\right],\left[p \cos \omega\left(\frac{q^{2}+p^{2}}{2}\right) t\right.\right. \\
& \left.\left.+q \sin \omega\left(\frac{q^{2}+p^{2}}{2}\right) t\right]\right\} .
\end{aligned}
$$

One has the example of classical f-oscillator, the so-called classical qoscillator $[5,6]$, for which

$$
f\left(\alpha \alpha^{*}\right)=\sqrt{\frac{\sinh \lambda \alpha \alpha^{*}}{\lambda \alpha \alpha^{*}}}, q=e^{\lambda} .
$$


For small nonlinearity $\lambda \ll 1$, one has the approximate value of the nonlinearity function

$$
f\left(\alpha \alpha^{*}\right) \simeq 1+\frac{\lambda^{2}}{12}\left(\alpha \alpha^{*}\right)^{2}=1+\frac{\lambda^{2}}{12} E^{2}, \quad E=\alpha \alpha^{*}
$$

and, in this case, the frequency of the q-oscillator is

$$
\omega(E)=1+\frac{\lambda^{2}}{4} E^{2}
$$

so it depends quadratically, for small nonlinearities, on the energy of vibrations.

\subsection{Quantum nonlinear oscillator}

Let us consider the quantum oscillator annihilation and creation operators $\hat{a}$ and $\hat{a}^{\dagger}$, respectively. For Hamiltonian of the form

$$
\hat{H}=\hat{a}^{\dagger} \hat{a}+\frac{1}{2},\left[\hat{a}, \hat{a}^{\dagger}\right]=\hat{1}
$$

one has the solution to Heisenberg evolution equation $(\hbar=1)$

$$
\hat{a}(t)=\hat{a} e^{-i t}, \quad \hat{a}=\frac{1}{\sqrt{2}}(\hat{q}+i \hat{p}),
$$

where $\hat{q}$ and $\hat{p}$ are the position and momentum operators, respectively.

Let us now consider the quantum f-oscillator with the Hamiltonian

$$
\hat{H}(\hat{n})=\frac{1}{2}\left(\hat{A}_{f}^{\dagger} \hat{A}_{f}+\hat{A}_{f} \hat{A}_{f}^{\dagger}\right), \quad \hat{n}=\hat{a}^{\dagger} \hat{a},
$$

where

$$
\hat{A}_{f}=\hat{a} f\left(\hat{a}^{\dagger} \hat{a}\right) .
$$

Now we consider the Hamiltonian of the form

$$
\hat{H}=\hat{A}_{f}^{\dagger} \hat{A}_{f}
$$

Physical phenomenon where the quantum f-oscillator Hamiltonian might prove to be useful is the Kerr effect (see, for instance, [41]). The description 
of such a system has been recently considered in [42] with the Hamiltonian (in dimensionless units)

$$
\hat{H}_{K}=\chi\left(\hat{a}^{\dagger}\right)^{2} \hat{a}^{2}+\hat{a}^{\dagger} \hat{a}
$$

where the light frequency is equal to unity and the crystal nonlinear susceptibility is $\chi$, with the aim of describing the evolution in terms of the Moyal representation. The above operator can be easily written as a function of the number operator $\hat{n}=\hat{a}^{\dagger} \hat{a}$,

$$
\hat{H}_{K}=\hat{n}+\chi \hat{n}(\hat{n}-1) .
$$

This suggests a description by f-oscillators introducing

$$
\hat{A}_{K}=\hat{a} f_{K}(\hat{n})
$$

with

$$
f_{K}(\hat{n})=\sqrt{1-\chi+\chi \hat{n}} .
$$

The function $f_{K}$ above introduced allows one to consider $f_{K}$-nonlinear coherent states and their evolution.

The f-oscillator for both Hamiltonians (28) and (30) has two time-dependent integrals of motion of the following form:

$$
\hat{Q}(t)=e^{-i \hat{H}(\hat{n}) t} \hat{a} e^{i \hat{H}(\hat{n}) t}, \quad \hat{Q}^{\dagger}(t)=e^{-i \hat{H}(\hat{n}) t} \hat{a}^{\dagger} e^{i \hat{H}(\hat{n}) t} .
$$

The total time derivative of these operators equals zero. For time $t=0$, the integrals of motion coincide with the annihilation and creation operators, respectively.

Due to the structure of the f-oscillator Hamiltonian, the eigenstates $\mid n, f>$ of this oscillator Hamiltonian coincide with the eigenstates of the operator $\hat{a}^{\dagger} \hat{a} \mid n>$ related to the usual oscillator. Since the Hamiltonian is expressed in terms of number operator $\hat{n}$, the result of commutation provides an explicit form of the integrals of motion. One can use the following formulae:

$$
\hat{a} \varphi(\hat{n})=\varphi(\hat{n}+\hat{1}) \hat{a}, \quad \hat{a}^{\dagger} \varphi(\hat{n}+\hat{1})=\varphi(\hat{n}) \hat{a}^{\dagger}
$$

where $\varphi(\hat{n})$ is an arbitrary function. Then the Hamiltonian (28) takes the form

$$
\hat{H}(\hat{n})=\frac{1}{2}\left(\hat{n} f(\hat{n})^{2}+(\hat{n}+1) f(\hat{n}+1)^{2}\right) .
$$


The above integrals of the motion can be written as

$$
\hat{Q}(t)=\hat{a} F(\hat{n}, t), \quad \hat{Q}^{\dagger}(t)=F(\hat{n}, t) \hat{a}^{\dagger},
$$

where the function $F(\hat{n}, t)$ for both Hamiltonians (28) and (30) reads

$$
F(\hat{n}, t)=f(\hat{n}) \exp [i\{\hat{H}(\hat{n})-\hat{H}(\hat{n}-\hat{1})\} t] .
$$

The evolution of any initial density state $\rho_{0}\left(\hat{a}, \hat{a}^{\dagger}\right)$ is given as

$$
\rho\left(\hat{a}, \hat{a}^{\dagger}, t\right)=\rho_{0}\left(\hat{Q}(t), \hat{Q}^{\dagger}(t)\right) .
$$

The above density operator satisfies the von Neumann evolution equation

$$
\frac{\partial}{\partial t} \rho+i[\hat{H}(\hat{n}), \rho]=0 .
$$

\section{$3 \quad$ Wigner functions}

The Wigner function of the evolving f-oscillator state reads

$$
W(q, p, t)=2 \operatorname{Tr}\left[\hat{P} \rho(t) \exp \left\{2\left(\alpha \hat{a}^{\dagger}-\alpha^{*} \hat{a}\right)\right\}\right],
$$

where $\hat{P}$ is the parity operator $\hat{P}=(-1)^{\hat{a}^{\dagger} \hat{a}}$ and complex number $\alpha$ is expressed in terms of the position and momentum as $\alpha=(q+i p) / \sqrt{2}$. The parity operator commutes with the Hamiltonian. In view of this, the Wigner function takes the form

$$
W(q, p, t)=2 \operatorname{Tr}\left[\hat{P} \rho_{0} \exp \left\{2\left(\alpha \hat{Q}^{\dagger}(t)-\alpha^{*} \hat{Q}(t)\right)\right\}\right] .
$$

The Wigner function (42) is the solution to the Moyal evolution equation [25] for the f-oscillator. To calculate explicitly the Wigner function, one needs to evaluate the trace in Eq. (38). It is worth noting that in Eq. (42) the Weyl system operator has the form of f-deformed shift operator which was used in [19]. The deformation function is given by Eq. (38).

When the initial state is the ground oscillator state $\rho_{0}=|0><0|$, one has the Wigner function

$$
W_{0}(q, p, t)=2<0\left|\exp \left[2\left(\alpha \hat{Q}^{\dagger}(t)-\alpha^{*} \hat{Q}(t)\right)\right]\right| 0>.
$$


Thus the Wigner function is proportional to the expectation value of the deformed shift operator in the vacuum state.

Let us now discuss the deformed creation and annihilation operators $\hat{A}_{f}^{\dagger}$ and $\hat{A}_{f}$ and the corresponding deformed parity operator, more formally.

The replacement of the usual boson operators $\hat{a}^{\dagger}$ and $\hat{a}$ by the deformed ones gives the deformation of the shift operator

$$
\exp \left[\left(\alpha \hat{a}^{\dagger}-\alpha^{*} \hat{a}\right)\right] \rightarrow \exp \left[\left(\alpha \hat{A}_{f}^{\dagger}-\alpha^{*} \hat{A}_{f}\right)\right],
$$

and the deformation of parity operator

$$
(-1)^{\hat{a}^{\dagger} \hat{a}}=\exp \left(i \pi \hat{a}^{\dagger} \hat{a}\right) \rightarrow \exp \left(i \pi \hat{A}_{f}^{\dagger} \hat{A}_{f}\right) .
$$

Then one has the possibility of introducing deformed Wigner functions using f-deformation of star-product quantization scheme. Namely, one gets either

$$
W_{f}^{(1)}(q, p)=2 \operatorname{Tr}\left[\hat{P} \hat{\rho} \exp \left\{2\left(\alpha \hat{A}_{f}^{\dagger}-\alpha^{*} \hat{A}_{f}\right)\right\}\right],
$$

keeping the parity operator in the usual form or

$$
W_{f}^{(2)}(q, p)=2 \operatorname{Tr}\left[\exp \left(i \pi \hat{A}_{f}^{\dagger} \hat{A}_{f}\right) \hat{\rho} \exp \left\{2\left(\alpha \hat{A}_{f}^{\dagger}-\alpha^{*} \hat{A}_{f}\right)\right\}\right] .
$$

For small deformations, the Wigner functions we have introduced do not differ much from the standard Wigner quasidistributions. The evolution in time of the deformed Wigner functions can be obtained by using the integrals of motion (37).

\section{Symplectic tomography}

Let us discuss in this section symplectic tomography [20] of classical states and nonlinear quantum states.

We will focus on symplectic tomograms of f-oscillator states.

Given a density state $\rho$, the symplectic tomogram also called tomographic symbol of the density operator is defined as

$$
w(X, \mu, \nu)=\operatorname{Tr}[\rho \delta(X \hat{1}-\mu \hat{q}-\nu \hat{p})],
$$

where $X, \mu, \nu$ are real numbers while $\hat{1}, \hat{q}$ and $\hat{p}$ are identity, position and momentum operators, respectively. In terms of the Wigner function, the tomogram reads

$$
w(X, \mu, \nu)=\int W(q, p) \delta(X-\mu q-\nu p) \frac{d q d p}{2 \pi} .
$$


The tomogram is a probability distribution function of the position $X$, i.e.,

$$
w(X, \mu, \nu) \geq 0
$$

and

$$
\int w(X, \mu, \nu) d X=1
$$

The parameters $\mu=s \cos \theta$ and $\nu=s^{-1} \sin \theta$ correspond to a reference frame in the phase space which is scaled $\left(q \rightarrow s q, p \rightarrow s^{-1} p\right)$ and thereafter rotated $\left(s q \rightarrow q s \cos \theta+p s^{-1} \sin \theta\right)$.

In classical mechanics, an analog of the tomographic symbol can be constructed for an arbitrary observable $\mathcal{A}(q, p)$, which is a function on the phase space, as the Radon integral

$$
w_{\mathcal{A}}(X, \mu, \nu)=\int \mathcal{A}(q, p) \delta(X-\mu q-\nu p) d q d p .
$$

Let us discuss now the tomographic representation for the classical f-oscillator. The solution to the Liouville equation (15) has the following tomogram for the nonlinear-oscillator state:

$$
\begin{aligned}
& w(X, \mu, \nu, t)=\int f_{0}\left\{\left[-p \sin \omega\left(\frac{q^{2}+p^{2}}{2}\right) t\right.\right. \\
& \left.\left.+q \cos \omega\left(\frac{q^{2}+p^{2}}{2}\right) t\right],\left[p \cos \omega\left(\frac{q^{2}+p^{2}}{2}\right) t+q \sin \omega\left(\frac{q^{2}+p^{2}}{2}\right) t\right]\right\} \\
& \times \delta(X-\mu q-\nu p) d q d p .
\end{aligned}
$$

The integral provides the solution (the tomogram) in terms of initial value of the tomogram $w_{0}(X, \mu, \nu)$ which is given as

$$
w_{0}(X, \mu, \nu)=\int f_{0}(q, p) \delta(X-\mu q-\nu p) d q d p .
$$

The symplectic tomogram of the f-oscillator quantum states is given in terms of the density operator (39) as

$$
w(X, \mu, \nu, t)=\operatorname{Tr}\left[\rho_{0}\left(\hat{Q}(t), \hat{Q}^{\dagger}(t)\right) \delta(X-\mu \hat{q}-\nu \hat{p})\right] .
$$

The quantum tomogram provides the probability distribution of the foscillator position $w(x, 1,0)=P(x)$ and the f-oscillator momentum $w(p, 0,1)=$ $\mathcal{P}(p)$. 
The ground state of the nonlinear oscillator satisfies the equation $A_{f} \mid 0, f>=$ 0 , which also satisfies the equation for the usual oscillator ground state $a \mid 0, f>=0$. This means that the tomogram of the f-oscillator ground state reads

$w_{0}(X, \mu, \nu)=\operatorname{Tr}[|0, f><0, f| \delta(X-\mu \hat{q}-\nu \hat{p})]=\frac{1}{\sqrt{\pi\left(\mu^{2}+\nu^{2}\right)}} \exp \left(-\frac{X^{2}}{\mu^{2}+\nu^{2}}\right)$.

The excited states of the nonlinear f-oscillator $\mid n, f>$ have the tomogram, which also coincides with the tomogram of the excited state $\mid n>$ of the usual harmonic oscillator, which reads

$$
w_{n}(X, \mu, \nu)=w_{0}(X, \mu, \nu) \frac{1}{2^{n} n !} H_{n}^{2}\left(\frac{X^{2}}{\sqrt{\mu^{2}+\nu^{2}}}\right) .
$$

The constructions of Wigner functions and tomograms of of the nonlinear oscillator's quantum states uses the operators (dequantizers) which are ingredients of star-product quantization schemes. Thus we found the symplectic tomograms of the basis excited states for both harmonic and nonlinear oscillators which turn out to be the same probability distributions.

\section{5 f-oscillator coherent states}

In $[1,2]$ the notion of f-oscillator nonlinear coherent state was introduced (see also [3]). The states are constructed as eigenstates of the operator $\hat{A}_{f}=$ $\hat{a} f(\hat{n})$, with $\hat{n}=\hat{a}^{\dagger} \hat{a}$ and the equation

$$
\hat{A}_{f}|\alpha, f>=\alpha| \alpha, f>
$$

where $\alpha$ is a complex number. For $f(\hat{n})=1$, the nonlinear coherent state coincides with usual coherent state $\mid \alpha>$, i.e.,

$$
\left|\alpha>=\exp \left[-\frac{|\alpha|^{2}}{2}\right] \sum_{n=0}^{\infty} \frac{\alpha^{n}}{\sqrt{n !}}\right| n>
$$

and $\hat{a}^{\dagger} \hat{a}|n>=n| n>, \quad n=0,1,2, \ldots$

The nonlinear coherent state is expressed as the following series in the Fock number states $\mid n>$

$\left|\alpha, f>=N_{f} \sum_{n=0}^{\infty} \frac{\alpha^{n}}{f(n) ! \sqrt{n !}}\right| n>, \quad f(n) !=f(0) f(1) f(2) \ldots f(n-1) f(n)$, 
where the normalization is given by the series

$$
N_{f}=\left(\sum_{n=0}^{\infty} \frac{|\alpha|^{2 n}}{[f(n) !]^{2} n !}\right)^{-1 / 2} .
$$

In the position representation, the nonlinear coherent state reads

$$
\psi_{\alpha, f}(x)=N_{f} \sum_{n=0}^{\infty} \frac{\alpha^{n}}{f(n) ! \sqrt{n !}}\left[e^{-\frac{x^{2}}{2}} \frac{1}{\pi^{1 / 4} \sqrt{n ! 2^{n}}} H_{n}(x)\right] .
$$

One can introduce two-mode nonlinear coherent states of the two-dimensional f-oscillator, for example, from operators

$$
\hat{A}_{1}=\hat{a}_{1} f\left(\hat{n}_{1}, \hat{n}_{2}\right) \quad \text { and } \quad \hat{A}_{2}=\hat{a}_{2} f\left(\hat{n}_{1}, \hat{n}_{2}\right),
$$

where $\hat{a}_{1}$ and $\hat{a}_{2}$ are annihilation operators for linear oscillators and $\hat{n}_{i}=\hat{a}_{i}^{\dagger} \hat{a}_{i}$, $i=1,2$. Their eigenstates are defined by the equations

$$
\hat{A}_{i}\left|\alpha_{1} \alpha_{2}, f>=\alpha_{i}\right| \alpha_{1} \alpha_{2}, f>\text {. }
$$

The nonlinearity is coded by the function of two variables $f\left(\hat{n}_{1}, \hat{n}_{2}\right)$ which, in fact, depends on the energies of both mode vibrations.

As an example, let us consider the nonlinearity given by the one variable function $f\left(\hat{n}_{1}+\hat{n}_{2}\right)$ where the coherent states are entangled by construction. The explicit form of such nonlinear oscillator coherent state in the position representation reads

$$
\Psi_{\alpha_{1} \alpha_{2} f}(x, y)=N_{f} \sum_{n_{1} n_{2}=0}^{\infty} \frac{\alpha_{1}^{n_{1}} \alpha_{2}^{n_{2}}}{\sqrt{n_{1} ! n_{2} !} f\left(n_{1}+n_{2}\right) !}<x y \mid n_{1} n_{2}>
$$

The normalization constant is given by the expression

$$
N_{f}=\left(\sum_{n_{1} n_{2}=0}^{\infty} \frac{\left|\alpha_{1}\right|^{2 n_{1}}\left|\alpha_{2}\right|^{2 n_{2}}}{n_{1} ! n_{2} !\left[f\left(n_{1}+n_{2}\right) !\right]^{2}}\right)^{-1 / 2}
$$

and

$$
<x y \mid n_{1} n_{2}>=\frac{e^{-\frac{x^{2}}{2}-\frac{y^{2}}{2}}}{\sqrt{\pi} \sqrt{2^{n_{1}+n_{2}} n_{1} ! n_{2} \mid}} H_{n_{1}}(x) H_{n_{2}}(y) .
$$


The functions $H_{n_{i}}$ are Hermite polynomials. For $f=1$, the nonlinear coherent state becomes separable two-mode coherent state

$\Psi_{\alpha_{1} \alpha_{2}}(x, y)=\frac{e^{-\frac{x^{2}}{2}-\frac{y^{2}}{2}}}{\sqrt{\pi}} \exp \left[-\frac{\left|\alpha_{1}\right|^{2}}{2}-\frac{\left|\alpha_{2}\right|^{2}}{2}+\sqrt{2}\left(\alpha_{1} x+\alpha_{2} y\right)-\frac{\alpha_{1}^{2}}{2}-\frac{\alpha_{2}^{2}}{2}\right]$

Let us discuss the introduced entanglement in more details.

The pure state of two-mode system is separable if its wave function is factorized, namely,

$$
\Psi\left(x_{1}, x_{2}\right)=\psi_{1}\left(x_{1}\right) \psi_{2}\left(x_{2}\right),
$$

where we denote the modes coordinates as $x_{1}$ and $x_{2}$. A wave function, which is the superposition of separable states, is entangled.

The usual nondeformed two-mode coherent states are separable. In fact,

$$
\left|\alpha_{1} \alpha_{2}>=\right| \alpha_{1}>\left|\alpha_{2}>\rightarrow<x_{1} x_{2}\right| \alpha_{1} \alpha_{2}>=<x_{1}\left|\alpha_{1}><x_{2}\right| \alpha_{2}>
$$

where

$$
<x_{1} \mid \alpha_{1}>=\exp \left[-\frac{\left|\alpha_{1}\right|^{2}}{2}\right] \sum_{n_{1}=0}^{\infty} \frac{\alpha_{1}^{n_{1}} e^{-\frac{x_{1}^{2}}{2}}}{\pi^{1 / 4} \sqrt{n_{1} !} \sqrt{2^{n_{1}} n_{1} !}} H_{n_{1}}\left(x_{1}\right)
$$

and

$$
<x_{2} \mid \alpha_{2}>=\exp \left[-\frac{\left|\alpha_{2}\right|^{2}}{2}\right] \sum_{n_{2}=0}^{\infty} \frac{\alpha_{2}^{n_{2}} e^{-\frac{x_{2}^{2}}{2}}}{\pi^{1 / 4} \sqrt{n_{2} !} \sqrt{2^{n_{2}} n_{2} !}} H_{n_{2}}\left(x_{2}\right)
$$

The nonlinear coherent states which we have introduced have the structure

$$
\left|\alpha_{1} \alpha_{2}, f>=N_{f} \sum_{n_{1} n_{2}=0}^{\infty} \frac{\alpha_{1}^{n_{1}} \alpha_{2}^{n_{2}}}{\sqrt{n_{1} ! n_{2} !} f\left(n_{1}+n_{2}\right) !}\right| n_{1} n_{2}>.
$$

In the case of $f\left(n_{1}+n_{2}\right)=1$, this series is reduced to the product of two series, each of them depending on single variable. But this is not the case in presence of the nonlinear function $f$ and therefore the nonlinearity creates entanglement. 


\section{Thermodynamics of f-oscillators}

For standard harmonic oscillators with Hamiltonian (26), the thermodynamical properties are determined by the partition function

$$
Z_{0}(\beta)=\operatorname{Tr} e^{-\beta \hat{H}}=\sum_{n=0}^{\infty} e^{-\beta(n+1 / 2)}=\frac{1}{2 \sinh (\beta / 2)} .
$$

The other characteristics are given using the partition function, like the energy

$$
\begin{aligned}
& E=\operatorname{Tr}\left(\hat{H} \frac{e^{-\beta \hat{H}}}{Z_{0}(\beta)}\right)=\frac{1}{Z_{0}(\beta)} \sum_{n=0}^{\infty}\left(n+\frac{1}{2}\right) e^{-\beta(n+1 / 2)} \\
& =\frac{1}{2}+\frac{1}{Z_{0}(\beta)}\left(-\frac{\partial}{\partial \beta} Z_{0}(\beta)\right)
\end{aligned}
$$

and the entropy

$$
\begin{aligned}
& S=-\operatorname{Tr}\left(\frac{e^{-\beta \hat{H}}}{Z_{0}(\beta)} \log \frac{e^{-\beta \hat{H}}}{Z_{0}(\beta)}\right) \\
& =-\frac{1}{Z_{0}(\beta)} \sum_{n=0}^{\infty} e^{-\beta(n+1 / 2)}\left[-\beta\left(n+\frac{1}{2}\right)-\log Z_{0}(\beta)\right] \\
& =\beta E+\log Z_{0}(\beta),
\end{aligned}
$$

which gives

$$
E-T S=-T \log Z_{0}(\beta),
$$

which is the free energy.

To consider the thermodynamics for f-oscillators, let us use the Hamiltonian $\hat{H}_{f}=\hat{A}_{f}^{\dagger} \hat{A}_{f}+1 / 2$. The partition function for such a system will be denoted $Z_{f}(\beta)$. For the nonlinearity function containing a small deviation from the linear case, we assume the nonlinearity to be expressed as

$$
f(\hat{n}) \approx(1+g \psi(\hat{n}))
$$

where $g \ll 1$ and $\psi(\hat{n})$ is some function of number of vibrations, i.e., we assume

$$
\hat{A}_{f} \approx \hat{a}[1+g \psi(\hat{n})]
$$


Then the proposed Hamiltonian of nonlinear oscillator takes the form

$$
\hat{H}_{f}=\hat{A}_{f}^{\dagger} \hat{A}_{f}+\frac{1}{2} \approx \hat{a}^{\dagger} \hat{a}+\frac{1}{2}+g \chi(\hat{n}),
$$

in which

$$
\chi(\hat{n})=2 \psi(\hat{n}) .
$$

Now one can calculate the partition function for this f-oscillator

$$
Z_{f}(\beta)=\operatorname{Tr} \exp \left[-\beta\left(\hat{a}^{\dagger} \hat{a}+\frac{1}{2}\right)-\beta g \chi(\hat{n})\right] .
$$

Keeping the same accuracy, i.e., linear terms in the small parameter $g$, we get

$$
Z_{f}(\beta)=\sum_{n=0}^{\infty} \exp \left[-\beta\left(n+\frac{1}{2}\right)\right][1-\beta g \chi(\hat{n})] .
$$

Repeating the calculations as done for the linear oscillator, we get the deformed partition function

$$
Z(\beta)_{f} \approx Z_{0}(\beta)[1-\beta g<\chi(\hat{n})>],
$$

where

$$
<\chi(\hat{n})>=\frac{1}{Z_{0}(\beta)} \sum_{n=0}^{\infty} \chi(n) e^{-\beta(n+1 / 2)} .
$$

Having the correction to the linear oscillator partition function, one can compute the small corrections to all the thermodynamical characteristics of the nonlinear f-oscillator, like free energy, entropy, etc.

For example, for quantum q-oscillator considered in [19]

$$
f(\hat{n})=\sqrt{\frac{\sinh \lambda \hat{n}}{\lambda \hat{n}}} \approx 1+\frac{\lambda^{2}}{12} \hat{n}^{2},
$$

we have

$$
\chi(\hat{n})=\frac{\lambda^{2}}{6} \hat{n}^{2} .
$$

Here $\lambda$ is a small parameter related to $g$, i.e., $g=\lambda^{2} / 6$. So in the present case, we have

$$
<\chi(\hat{n})>=<\hat{n}^{2}>=\frac{1}{Z_{0}(\beta)} \sum_{n=0}^{\infty} n^{2} e^{-\beta(n+1 / 2)}=\frac{1}{2} \operatorname{coth} \frac{\beta}{2}\left(\operatorname{coth} \frac{\beta}{2}-1\right) .
$$


Thus the correction for the partition functions for small q-oscillator nonlinearity reads

$$
Z(\beta)_{f}-Z_{0}(\beta) \approx-\frac{g \beta}{2} \operatorname{coth} \frac{\beta}{2}\left(\operatorname{coth} \frac{\beta}{2}-1\right)
$$

For large temperature $\beta \rightarrow 0$, the thermodynamic characteristics of the nonlinear quantum oscillator become the thermodynamic characteristics of the classical q-oscillator.

\section{Conclusions}

To conclude, we point out the main results of our work.

We studied nonlinear f-oscillators in both classical and quantum settings and found new time-dependent integrals of motion for the oscillators. For both settings, we obtained the solutions to the kinetic equations like Liouville classical equation for probability distribution on the phase space and von Neumann equation for the density operator of the f-oscillator states. Using the deformation function determining the f-oscillator, we introduced the deformed Wigner functions by deforming Weyl displacement operator in the formalism of Moyal star-product quantization. Also we studied symplectic tomography of the f-oscillator states and obtained the tomographic probability of the ground and excited states of the f-oscillators, which were shown to coincide with the tomograms of the excited states of the linear harmonic oscillator. We showed that the particular nonlinearity of vibrations we have considered in the case of two-mode nonlinear coherent states has created entangled states. We calculated the thermodynamic properties of the nonlinear quantum oscillators in the case of small nonlinearity and found explicit corrections to the partition function of the linear oscillator induced by small nonlinearity of q-oscillator. We showed that known Kerr nonlinearity of media can be considered within the framework of a specific nonlinear oscillator formalism.

\section{Acknowledgements}

VIM thanks Dipartimento di Scienze Fisiche, Università "Federico II" di Napoli and Istituto Nazionale di Fisica Nucleare, Sezione di Napoli for kind 
hospitality and the Russian Foundation for Basic Research for a partial support under Projects Nos. 07-02-00598 and 09-02-00142.

\section{References}

[1] Man'ko V I, Marmo G, Sudarshan E C G and Zaccaria F 1997 Phys. Scr. 55528

[2] Man'ko V I, Marmo G, Sudarshan E C G and Zaccaria F 1996 foscillators Proc. IV Wigner Symp. (Guadalajara, Mexico, July 1995) ed N M Atakishiyev, T M Seligman and K-B Wolf (Singapore: World Scientific ) p 421

[3] de Matos Filho R L and Vogel W 1994 Phys. Rev. A 492812 de Matos Filho R L and Vogel W 1996 Phys. Rev. A 544560

[4] Man'ko V I, Marmo G, Solimeno S and Zaccaria F 1993 Phys. Lett. A 176173

[5] Biedenharn L C 1989 J. Phys. A: Math. Gen. 22 L873

[6] Macfarlane A J 1989 J. Phys. A: Math. Gen. 221581

[7] Darareh M D, Naderi M H and Soltanolkotabi M 2009 Opt. Commun. 2824577

[8] Popov D, Pop N, Luminosu I, et al. 2009 Eur. Phys. Lett. 8744003

[9] Ben Geloun J and Hounkonnou M N 2009 J. Phys. A: Math. Theor. 42 295202

[10] Shchukin E, Kiesel T and Vogel W 2009 Phys. Rev. A 79043831

[11] Castaneda J A, Hernandez M A and Jauregui R 2008 Phys. Rev. A 79 023809

[12] Ali S T, Gazeau J P and Heller B 2008 J. Phys. A: Math. Gen. 41365302

[13] Recamier J, Gorayeb M, Mocha W L, et al. 2008 J. Theor. Phys. 47673

[14] Kastrup H A 2007 Ann. Phys. 16439 
[15] Dodonov V V and Reno M B 2006 J. Phys. A: Math. Gen. 397411

[16] Dell'Anno F, De Siena S and Illuminati F 2006 Phys. Rep. - Rev. Sec. Phys. Lett. 42853

[17] Pinzul A and Stern A 2005 Gauge theory of the star-product ArXiv: hep-th $0705.1785 \mathrm{v} 2$

[18] Man'ko V I and Mendes R V 1998 J. Phys. A: Math. Gen. 316037

[19] Aniello P, Man'ko V I, Marmo G, et al 2000 J. Opt. B: Quantum Semiclass. Opt. 2718

[20] Mancini S, Man'ko V I and Tombesi P 1996 Phys. Lett. A 2131

[21] Man'ko O V and Man'ko V I 1997 J. Russ. Laser Res. 18407

[22] Man'ko V I and Mendes R V 2000 Physica D 145330

[23] Radon J 1917 Ber. Sachs. Akad. Wiss., Leipzig 69262

[24] Wigner E P 1932 Phys. Rev. 40749

[25] Moyal J E 1949 Proc. Camb. Phil. Soc. 4599

[26] Weyl H 1927 Z. Phys. 481

[27] Man'ko O V, Man'ko V I and Marmo G 2002 J. Phys. A: Math. Gen. 35699

[28] Man'ko O V, Man'ko V I, Marmo G and Vitale P 2007 Phys. Lett. A 360522

[29] Man'ko V I, Marmo G, Simoni A and Ventriglia F 2006 Open Syst. Inform. Dyn. 13239

[30] Man'ko V I, Marmo G, Simoni A et al. 2006 Phys. Lett. A 3511

[31] Ibort A, Man'ko V I, Marmo G et al. 2009 Phys. Scr. 79065013

[32] Man'ko O V, Man'ko V I and Marmo G 2000 Phys. Scr. 62446

[33] Man'ko V I, Marmo G and Vitale P 2005 Phys. Lett. A 3341 
[34] Man'ko V I, Marmo G, Vitale P and Zaccaria F 1994 Int. J. Mod. Phys. A 95541

[35] Zachos C 2002 Int. J. Mod. Phys. A 17297

[36] Bayen F, Flato M, Fronsdal C et al. 1978 Ann. Phys. NY 11161

[37] Berezin F A 1975 Commun. Math. Phys. 40153

[38] Baker Jr. G A 1958 Phys. Rev. 1092198

[39] Stratonovich R L 1956 Zh. Éksp. Teor. Fiz. 311012

[40] Dodonov V V and Man'ko V I 1987 Invariants and the Evolution of Nonstationary Quantum Systems Proceedings of the Lebedev Physical Institute, Nauka, Moscow, Vol. 183 [translated by Nova Science, New York, 1989].

[41] Mandel L and Wolf E 1995 Optical Coherence and Quantum Optics, Cambridge University Press, p. 1102

[42] Osborn T A and Marzlin K-P 2009 Moyal phase-space analysis of nonlinear optical Kerr media ArXiv/0905.3530v1 